\title{
Pearls \& Oy-sters: A curable myopathy manifesting as exercise intolerance and respiratory failure
}

André M.S. Silva, MD, Rodrigo H. Mendonça, MD, Diogo C. Soares, MD, Dagoberto Callegaro, MD, PhD, Vitor M. Caldas, MD, lago N. Perissinotti, MD, Mary S. Carvalho, MD, PhD, and Edmar Zanoteli, MD, PhD

Neurology ${ }^{\circledR}$ 2018;91:187-190. doi:10.1212/WNL.0000000000005867

\section{Pearls}

- $\quad$ Few forms of severe genetic myopathies can successfully be treated, although multiple acylCoA dehydrogenase deficiency (MADD) is highly responsive to riboflavin supplementation.

- Muscle MRI and muscle biopsy play important roles in patients with exercise intolerance and dyspnea when metabolic myopathy is suspected.

\section{Oy-sters}

- EMG and serum creatine kinase (CK) levels may be normal in metabolic myopathies. Muscle biopsy may be the only abnormal ancillary examination.

- MADD must be considered in cases of rapidly progressive respiratory insufficiency, as this condition is potentially treatable.

\section{Case report}

A 32-year-old woman presented with a 2-year history of progressive exercise intolerance and lower limb myalgia. She noted progressive difficulty in walking long distances and climbing stairs as well as dyspnea. No relevant familial history or consanguinity was reported. The patient's motor development was unremarkable.

In the first neurologic evaluation, mild weakness was noted (Medical Research Council [MRC] 4 in the upper and lower limbs, proximal and distal segments, as well in the neck muscles). The cranial nerve examination was normal. The patient's limitation was mainly due to minimal effort intolerance and resistance to examination maneuvers, making her motor examination difficult. Deep tendon reflexes and sensory examination were normal. Forced vital capacity (FVC) and forced expiratory volume in 1 second were reduced (52\% and 57\%, respectively), indicating a possibly restrictive disorder, which is nonspecific. Several serum CK measures, echocardiography, chest radiography, thorax CT, abdominal ultrasound, and EMG/nerve conduction study (NCS) were normal. The patient's serum acylcarnitine profile was unremarkable. A dry blood spot screening for Pompe disease was negative. As all ancillary examinations were normal, the patient was referred to a psychiatrist, but no mental abnormality was detected.

As the patient remained with complaints of fatigue and muscle pain, and a suspicion of metabolic myopathy existed, a noninvasive test was initially used to detect any objective skeletal muscle involvement. A muscle MRI was obtained and depicted a mild fatty infiltration in the posterior compartment of the thighs without muscle edema or gadolinium enhancement (figure, A). After this finding, a muscle biopsy from the left biceps brachii
Correspondence

Dr. Silva

andre.macedo@hc.fm.usp.br 

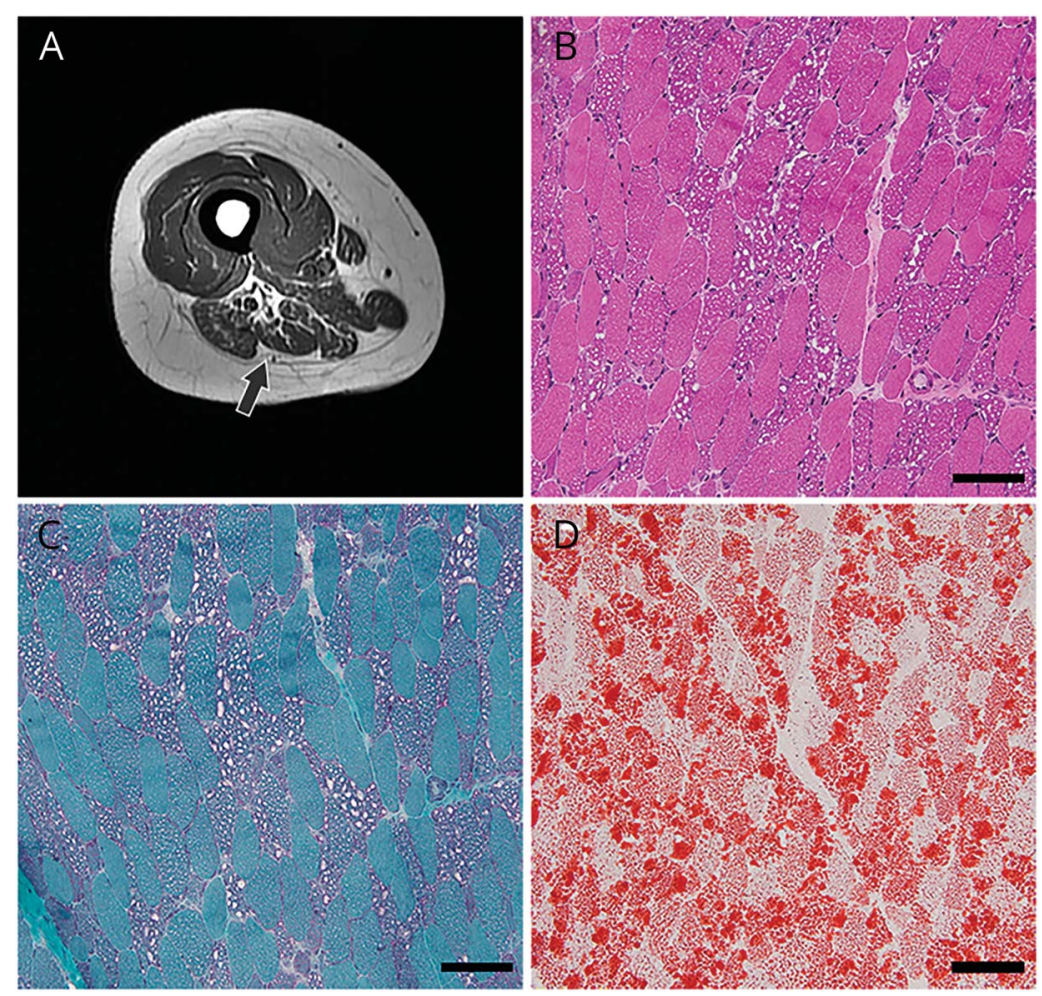

(A) Axial T1-weighted MRI of the thigh shows mild fatty infiltration in posterior compartment (arrow). (B, C) Biceps brachii muscle biopsy reveals a myopathic aspect with intracytoplasmic vacuoles on hematoxylin \& eosin and modified Gomori trichrome stainings. (D) Oil-Red-O staining confirms lipid accumulation in the vacuoles. Bar: $50 \mu \mathrm{m}$. was done, and it revealed a vacuolar myopathy with massive lipid droplets, predominantly in type 1 fibers (figure, B-D).

During the investigation period, the patient presented rapid deterioration of respiratory insufficiency requiring noninvasive mechanical ventilation, dysphagia, intense vomiting, weight loss, and a marked weakness with dropped head (MRC 2/5 in proximal limbs and axial muscles). After deterioration, a second EMG/NCS showed myopathic findings in the neck, deltoids, and thigh adductor muscles, and the CK level was mildly increased ( $343 \mathrm{U} / \mathrm{L}$, normal range 26-140 U/L).

Considering a probable diagnosis of lipid storage disease based on muscle biopsy and rapid clinical worsening, the patient received riboflavin, coenzyme Q10, and L-carnitine in high doses $(800 \mathrm{mg} / \mathrm{d}, 1,200 \mathrm{mg} / \mathrm{d}$, and $3,000 \mathrm{mg} / \mathrm{d}$, respectively), while whole exome sequencing was ordered. After 3 weeks of supplementation, she presented a dramatic recovery with normalization of the motor examination and FVC.

The whole exome sequencing identified compound heterozygous pathogenic mutations in the electron transfer flavoprotein dehydrogenase (ETFDH) gene, c.295C>T (p.Arg99Cys) and c.1547T >C (p.Phe516Ser), and the diagnosis of MADD was made. The patient continued receiving riboflavin $400 \mathrm{mg} / \mathrm{d}$ and coenzyme Q10 $400 \mathrm{mg} / \mathrm{d}$, and she displayed no symptoms at the 3-month follow-up.

\section{Discussion}

MADD, also known as glutaric aciduria type II, is an autosomal recessive inherited disorder of fatty and organic acid metabolism caused by mutations in 3 different genes (ETFA, $E T F B, E T F D H)$ involved in electron transfer to the respiratory chain by flavoproteins. ${ }^{1}$ MADD has a wide clinical presentation, from a neonatal-onset phenotype with severe acidosis, hypoglycemia, encephalopathy, and cardiomyopathy to an adult-onset milder presentation with exercise intolerance, cyclical vomiting, and progressive muscle weakness. $^{2}$

Some patients, like ours, with late-onset disease present rapid neurologic deterioration with severe proximal and axial weakness (with marked dropped head), dysphagia, vomiting, and respiratory insufficiency. ${ }^{2}$ However, for the previous 2 years, the main complaints of our patient were fatigue and mild dyspnea. The initial workup was unremarkable, including serum CK levels, acylcarnitine profile, and EMG, making her diagnosis difficult for different physicians. Both scenariossevere and mild presentation-are challenging, as MADD is an uncommon condition with unspecific symptoms, especially when laboratory examinations and electrodiagnostic 
testing are normal. ${ }^{3}$ Misdiagnoses with polymyositis and psychiatric disorder, as well as delay of precise diagnosis, are reported. $^{3,4}$

In our patient, the blood acylcarnitine profile was normal. This test is generally helpful when lipid metabolism disorder is suspected; it must be performed after at least a 12-hour fasting period, and it usually reveals the accumulation of all types of acylcarnitines (C4-C18). ${ }^{5}$ In addition, urinary organic acid analysis can display, in some patients, a combination of increased conjugate acids, typically with d-2-hydroxyglutaric aciduria, increased dicarboxylic acids, hexanoylglycine, and glycine conjugates excretion. ${ }^{1,5}$ However, some patients with MADD do not display typical biochemical patterns of urine organic acids and blood acylcarnitines, with levels being normal, especially if the test is undertaken when the patient is stable. ${ }^{6}$

The high suspicion for metabolic myopathy and the abnormal muscle MRI were decisive in performing the muscle biopsy in our case. Muscle MRI has become essential as a noninvasive step in the investigation of muscle diseases, sometimes supporting the decision to ask for a more invasive examination. ${ }^{7}$ In patients with MADD, fat infiltration has been demonstrated by $\mathrm{T} 1$-sequence MRI in the posterior compartment of the thighs, as noted in our patient, although this finding is not specific. $^{8}$

Two heterozygous missense mutations in the ETFDH gene were identified in our patient. Mutations in this gene are detected in most patients with MADD and were reported in 93\% of individuals in a large reviewed series of cases. ${ }^{6}$ The first variant (c.295C>T) promotes a substitution of arginine by cysteine in position 99 in exon 3, and this mutation has been previously described as pathogenic. ${ }^{9}$ The second variant $(\mathrm{c} .1547 \mathrm{~T}>\mathrm{C})$ is novel and leads to a substitution of phenylalanine by serine in position 516 in exon 12 . This variant was not previously described, is not present in large population databases (EXAC, GNoMED, EVS), and is not reported in the disease databases (ClinVar and HGMD). This mutation is present in a very conserved residue and is predicted to be damaging according to all prediction tools available in ANNOVAR ${ }^{10}$ Thus, the combination of molecular mechanism, characteristic of the region where the mutation is located, and the strong clinical correlation suggests this variant is probably pathogenic.

Metabolic myopathies include disorders of glycogen and glycolysis metabolism, known as glycogen storage diseases (GSD), lipid metabolism, and mitochondrial respiratory chain. Presentation may involve fluctuating symptoms, such as myalgia, cramps, exercise intolerance, or rhabdomyolysis, but some patients present with progressive weakness. ${ }^{11}$ Differentiating clinical carbohydrate metabolism disorder from lipid metabolism defects is challenging and the first step in the approach of metabolic myopathy.

Glycogenolysis and glycolysis are important during short exercise, and disorders of these pathways can manifest in 2 main presentation forms: as initial effort intolerance, myalgia, postexertional myoglobinuria, and episodes of CK increase as in McArdle disease (GSD type 5) and Tarui disease (GSD type 7 ), or presenting with progressive proximal muscle weakness associated with respiratory insufficiency as in lateonset Pompe disease (GSD type 2). ${ }^{12}$ These 3 diseases are the most common disorders of carbohydrate metabolism in clinical practice.

Fatty acids are the main energy source for muscle during prolonged exercise and fasting periods. Fatty acid oxidation dysfunction involves disorders with transient intracellular lipid accumulation with rhabdomyolysis, as carnitine palmitoyl transferase II (CPT II) deficiency, very-long-chain acylCoA dehydrogenase (VLCAD) deficiency, and mitochondrial trifunctional protein (MTP) deficiency, and another group of lipid storage disorders with fatigue, progressive weakness, and marked lipid droplets accumulation, as MADD, primary carnitine deficiency, neutral lipid storage disease with myopathy, and neutral lipid storage disease with ichthyosis. ${ }^{13}$

MADD is not the most common lipid metabolism disorder. CPT II, VLCAD, and MTP deficiencies are the most frequent causes, and their presentation predominantly involves episodes of rhabdomyolysis with transitory and mild or no lipid accumulation in the muscle fibers, and the muscle biopsy between acute episodes is often normal. ${ }^{11,13}$ However, in lipid storage disorders, as in MADD, the muscle analysis plays a fundamental role because it shows massive lipid storage, narrowing the differential diagnosis.

The approach of metabolic myopathy depends on the main presentation. If the symptoms evolve during short and moderate to intense exercises, as in isometric effort, the main hypotheses are McArdle and Tarui disease, and a forearm exercise test or a muscle biopsy to evaluate the enzyme activity is recommended, followed by gene testing for these conditions ( $P F K$ and PYGM genes). For patients with progressive limb-girdle weakness with respiratory failure, Pompe disease is considered, and leukocyte acid maltase enzyme activity in dry blood spot testing must be done followed by genetic confirmation (GAA gene). In cases of prolonged exercise intolerance and repeated rhabdomyolysis, serum acylcarnitines and urinary organic acids are useful to identify abnormalities in lipid metabolism and to direct the genetic testing for the main diseases as related to CTPII, VLCAD, MTP, and $E T F D H$ genes. If this triage for lipid accumulation is normal, a muscle biopsy that may reveal that lipid storage in MADD should be considered. ${ }^{11-13}$ In all cases, muscle MRI can be used as a noninvasive test to detect mild muscle involvement, and a whole exome may be requested in some cases, as in ours, because of institutional characteristics.

Among lipid metabolism disorders, the importance of diagnosing MADD comprises the favorable therapeutic response to riboflavin supplementation. The effect of riboflavin in this condition is not well-understood, but it is supposed 
that riboflavin plays a role by increasing intramitochondrial flavin adenine dinucleotide and stabilizing the mutant flavoprotein. ${ }^{14}$ In fact, our patient rapidly recovered from all neurologic symptoms after an abrupt life-threatening progression.

Hence, MADD must be considered in 2 adult settings: patients with progressive dyspnea with fatigue and exercise intolerance and cases of acute muscular deterioration with bulbar symptoms and axial involvement, such as dropped head syndrome. Among different diagnostic possibilities, the patient may have a curable inherited myopathy.

\section{Author contributions}

Dr. Silva: study concept, acquisition and analysis of data, literature review, and initial draft of the paper. Dr. Mendonça: acquisition and analysis of data, literature review, and critical revision of manuscript for intellectual content. Dr. Soares: acquisition and analysis of data, literature review, and critical revision of manuscript for intellectual content. Dr. Callegaro: acquisition of data, literature review, and critical revision of manuscript for intellectual content. Dr. Caldas: acquisition of data, literature review, and critical revision of manuscript for intellectual content. Dr. Perissinotti: acquisition of data, literature review, and critical revision of manuscript for intellectual content. Dr. Carvalho: study concept, acquisition of data, literature review, and critical revision of manuscript for intellectual content. Dr. Zanoteli: study concept, acquisition and analysis of data, literature review, and critical revision of manuscript for intellectual content.

\section{Study funding}

No targeted funding reported.

\section{Disclosure}

The authors report no disclosures relevant to the manuscript. Go to Neurology.org/N for full disclosures.

\section{References}

1. Olsen RK, Olpin SE, Andresen BS, et al. ETFDH mutations as a major cause of riboflavinresponsive multiple acyl-CoA dehydrogenation deficiency. Brain 2007;130:2045-2054

2. Ohkuma A, Noguchi S, Sugie H, et al. Clinical and genetic analysis of lipid storage myopathies. Muscle Nerve 2009;39:333-342.

3. Köppel S, Gottschalk J, Hoffmann GF, Waterham HR, Blobel H, Kölker S. Late-onset multiple acyl-CoA dehydrogenase deficiency: a frequently missed diagnosis? Neurology 2006;67:1519.

4. Vengalil S, Preethish-Kumar V, Polavarapu K, et al. Fatty acid oxidation defects presenting as primary myopathy and prominent dropped head syndrome. Neuromuscul Disord 2017;27:986-996.

5. Béhin A, Acquaviva-Bourdain C, Souvannanorath S, et al. Multiple acyl-CoA dehydrogenase deficiency (MADD) as a cause of late-onset treatable metabolic disease. Rev Neurol 2016;172:231-241.

6. Grünert SC. Clinical and genetical heterogeneity of late-onset multiple acyl-coenzyme A dehydrogenase deficiency. Orphanet J Rare Dis 2014;9:117.

7. Cardy CM, Potter T. The predictive value of creatine kinase, EMG and MRI in diagnosing muscle disease. Rheumatology 2007;46:1617-1618.

8. Liu XY, Jin M, Wang ZQ, et al. Skeletal muscle magnetic resonance imaging of the lower limbs in late-onset lipid storage myopathy with electron transfer flavoprotein dehydrogenase gene mutations. Chin Med J 2016;129:1425-1431.

9. Chien YH, Lee NC, Chao MC, et al. Fatty acid oxidation disorders in a Chinese population in Taiwan. JIMD Rep 2013;11:165-172.

10. Wang K, Li M, Hakonarson H. ANNOVAR: functional annotation of genetic variants from high-throughput sequencing data. Nucleic Acids Res 2010;38:e164.

11. Toscano A, Barca E, Musumeci O. Update on diagnostics of metabolic myopathies. Curr Opin Neurol 2017;30:553-562.

12. Lilleker JB, Keh YS, Roncaroli F, Sharma R, Roberts M. Metabolic myopathies a practical approach. Pract Neurol 2018;18:14-26.

13. Laforêt P, Vianey-Saban C. Disorders of muscle lipid metabolism: diagnostic and therapeutic challenges. Neuromuscul Disord 2010;20:693-700.

14. Zhu M, Zhu X, Qi X, et al. Riboflavin-responsive multiple Acyl-CoA dehydrogenation deficiency in 13 cases, and a literature review in mainland Chinese patients. J Hum Genet 2014;59:256-261.

\section{Visit the Neurology $y^{\circledR}$ Resident \& Fellow Website}

Click on Residents \& Fellows tab at Neurology.org.

Now offering:

- Neurology ${ }^{\circledR}$ Resident \& Fellow Editorial team information

- "Search by subcategory" option

- E-pearl of the Week

- RSS Feeds

- Direct links to Continuum ${ }^{\circledR}$, Career Planning, and AAN Resident \& Fellow pages

- Recently published Resident \& Fellow articles

- Podcast descriptions

f Find Neurology ${ }^{\circledR}$ Residents \& Fellows Section on Facebook: http://tinyurl.com/o8ahsys

Follow Neurology ${ }^{\circledR}$ on Twitter: http://twitter.com/GreenJournal 


\section{Neurology}

\section{Pearls \& Oy-sters: A curable myopathy manifesting as exercise intolerance and respiratory failure}

André M.S. Silva, Rodrigo H. Mendonça, Diogo C. Soares, et al.

Neurology 2018;91;187-190

DOI 10.1212/WNL.0000000000005867

This information is current as of July 23, 2018

\section{Updated Information \& Services}

References

Subspecialty Collections

\section{Permissions \& Licensing}

Reprints including high resolution figures, can be found at: http://n.neurology.org/content/91/4/187.full

This article cites 14 articles, 2 of which you can access for free at: http://n.neurology.org/content/91/4/187.full\#ref-list-1

This article, along with others on similar topics, appears in the following collection(s):

\section{All Neuromuscular Disease}

http://n.neurology.org/cgi/collection/all_neuromuscular_disease Lipidoses

http://n.neurology.org/cgi/collection/lipidoses

Metabolic disease (inherited)

http://n.neurology.org/cgi/collection/metabolic_disease_inherited Muscle disease

http://n.neurology.org/cgi/collection/muscle_disease

Organic acid

http://n.neurology.org/cgi/collection/organic_acid

Information about reproducing this article in parts (figures,tables) or in its entirety can be found online at:

http://www.neurology.org/about/about_the_journal\#permissions

Information about ordering reprints can be found online:

http://n.neurology.org/subscribers/advertise

Neurology ${ }^{\circledR}$ is the official journal of the American Academy of Neurology. Published continuously since 1951, it is now a weekly with 48 issues per year. Copyright () 2018 American Academy of Neurology. All rights reserved. Print ISSN: 0028-3878. Online ISSN: 1526-632X.

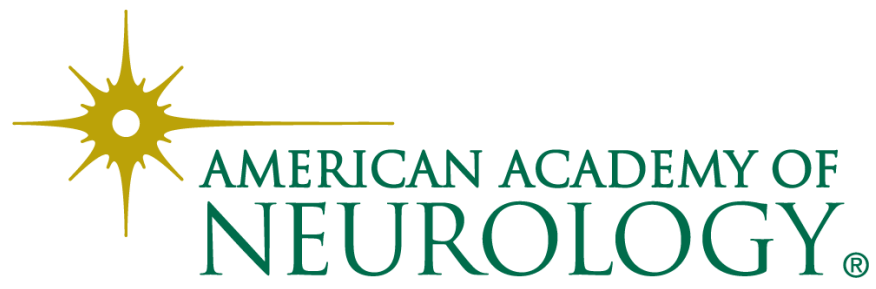

